Hackler, C., Byse, E., Alves, T.C.L., and Reed, D. (2019). "Building a Lean Culture: Engaging the Value Stream." In: Proc. 27th Annual Conference of the International. Group for Lean Construction (IGLC), Pasquire C. and Hamzeh F.R. (ed.), Dublin, Ireland, pp. 169-178. DOI: https://doi.org/10.24928/2019/0169. Available at: <www.iglc.net>.

\title{
BUILDING A LEAN CULTURE: ENGAGING THE VALUE STREAM
}

\author{
Cory Hackler ${ }^{1}$, Erika Byse ${ }^{2}$, Thais da C. L. Alves ${ }^{3}$, and Dean Reed ${ }^{4}$
}

\begin{abstract}
This paper presents an analysis of a Lean Leadership (LL) training program initiated by the company about three years ago. The program's main goal is to disseminate Lean throughout the company, which has been using Lean principles in its projects for about twenty years. So far, the LL program has reached over four hundred participants. Over the last year, the program included participants from the company's extended value stream. Participants include project teams and the company's strategic partners for prefabrication, equipment rental, and VDC/Project Controls support services. As part of the program, authors one and two visited participants to understand how they are applying lean leadership principles. This paper, the third in the series of building a Lean culture, shares success stories on how organizations in the company's value stream applied LL knowledge to their business including value stream mapping, Plan-Do-Check-Act (PDCA), go and see, and effective meetings. It also presents how these teams will continue their LL training to further build a Lean culture which the company can learn from its strategic partners while driving home a common purpose.
\end{abstract}

\section{KEYWORDS}

Lean leadership, training, Lean journey, change.

\section{INTRODUCTION}

A value stream can be defined as the combination of all value adding, non-value adding, and supporting activities necessary to deliver a product or service (Rother and Shook 1998). These activities are performed by multiple organizations, with different attributions, cultures, and values, in geographically dispersed supply chains. Faced with this reality, general contractors constantly work to align their project partners towards delivering value to clients. However, while project partnering sessions, design charrettes, and collaborative contracts, to name a few, support this endeavour, little is done to imprint long lasting changes on the way extended value streams work to deliver construction projects.

\footnotetext{
DPR Construction, 1450 Veterans Boulevard, Redwood City, CA. CoryHa@dpr.com DPR Construction, 222 N. 44th Street, Phoenix, Arizona 85034, ErikaB@dpr.com

Associate Professor, J.R. Filanc Construction Engineering and Management Program, Dept. of Civil, Constr., and Env. Engineering, San Diego State University, USA, talves@sdsu.edu

4 DPR Construction, 1450 Veterans Boulevard, Redwood City, CA. DeanR@dpr.com
} 
This paper presents an analysis of a current effort by a construction company to align its personnel regarding the use of Lean through the development of a Lean Leadership training program. The program was first discussed in a previous IGLC paper (Hackler et al. 2017), which explained the initial steps the company took in their journey to develop Lean leaders. The second paper focuses mainly on some lessons learned from the program, what participants seem to value in the training program, what can be improved in the future (Hackler et al. 2018). This paper focuses on extending the program to the value stream and the conclusion will address how to accelerate and measure the success including whole life integration.

\section{LEAN THINKING AND VALUE STREAMS}

The discussion about integrating construction supply chains and their related value streams took place in Lean Construction literature during the early 2000s with great intensity. Ballard and Howell (2003) outlined the different stages of the Lean Project Delivery System (LPDS) and highlighted the need to have them intersect with their immediate link; for instance, project definition decisions should intersect with the design process, which should overlap with the supply effort, which in turn should intersect with assembly, and that with the use phase. Along these lines, Tommelein et al. (2003) studied different supply chains and used value stream mapping to outline how construction supply chains work and how they can be made more efficient to deliver value.

Vrijhoef and Koskela (2000) defined the four roles of supply chain management in construction considering the following relationships between projects and their value streams: 1. Focus on the interface between the project and the supply chain; 2. Focus exclusively on the supply chain; 3 . Transfer site activities to the supply chain (e.g., through prefabrication); and 4. Focus on the integrated relationship between the project and its supply chain.

Alongside what is documented in the literature, the company is currently working to align its immediate project partners (Role 2) by using Lean principles and concepts outlined in the LPDS framework and supporting its partners to learn Lean and develop their own mapping and investigation efforts to better deliver value to its clients and stakeholders. The LL training supports these efforts, but it is not the only one currently being deployed at the company. Another concrete example, the company has been using to align its partners, focuses on delivering quality products by clearly defining a common language regarding what is expected from its value stream partners via Distinguishing Features of Work (DFOW), aligning the teams, agreeing on measurable criteria of acceptance, and verifying that the deliverables match the defined criteria (Spencley et al. 2018).

\section{CURRENT STATE OF THE LEAN LEADERSHIP TRAINING}

The cases presented in this paper are related to companies which are part of a Californiabased, top 50 United States-ranked general contractor's (GC) value stream. The GC currently has 26 offices in the United States, and three overseas. The GC started offering the LL training three years ago to its own personnel, and in the most recent edition in 2018, the course's ninety available seats filled up within twenty minutes, leaving an additional 
two hundred people on a wait list, and other business units are offering their own training, including the company's value stream partners. This demand serves as an indication of the value of the LL training, and the need to align a rapidly growing company with its evergrowing network of partners. While the program started with the intention to train employees within the company to better manage their work, it has evolved into one of the most important steps in aligning the company's extended value stream. This is the background of the cases presented and how they are related to the GC's value stream.

The diagram shown in figure 1 introduced by the authors (Hackler et al. 2018), illustrates the idea that stakeholders, who continue to learn and are exposed to the company's shared goals, values, and beliefs, become more aligned and begin to improve performance, as well as meet customer goals. This idea is now being applied by the company, through involving its strategic partners in the LL program, with the intention of developing and sharing common team goals throughout their value streams.

Figure 1: Leading with Purpose and Principles

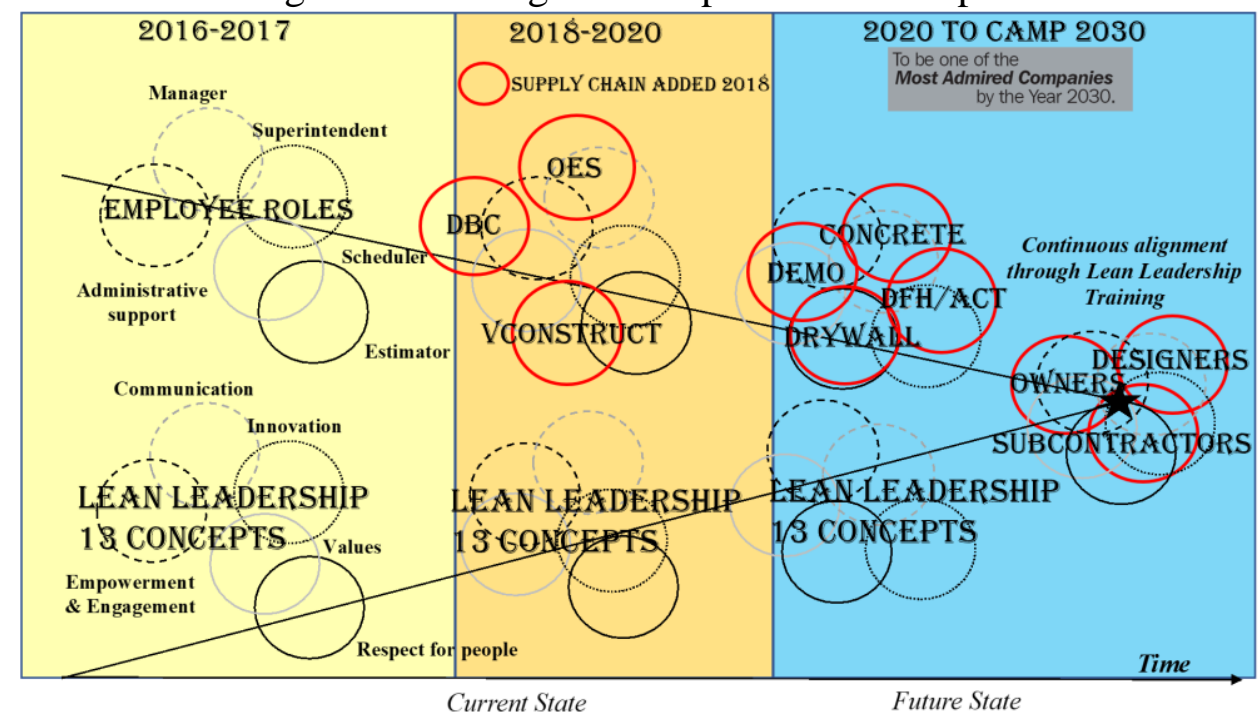

This paper presents four examples of value stream partners and their ability to apply LL concepts after taking the LL training. The stories address the journeys of the following:

1. A prefabrication company of exterior and interior walls that is less than four years old.

2. A Virtual Design and Construction (VDC) and Project Controls service company that has doubled their size in the last two years.

3. An equipment rental/supply company that has doubled their growth in the last three years.

4. A large IPD project in the Bay Area whose extended team took the LL training 


\section{RESEARCH APPROACH}

The method used to gather data was to "go and see" the locations of the affiliated value stream and have lean leaders at each company visited show authors 1 and 2 what they implemented based on their lean leadership training. Others in the organization were also interviewed to see if the lean leadership behaviour changes were trickling throughout the organization.

The data collection happened longitudinally through interviews, observations, and conversations with leaders of the companies visited, in addition to pictures taken to document the practices/examples encountered. Common threads/questions during the interviews were related to: how the partner/s took the training, what they implemented from the training, and the results achieved. The observations of each case, in each of the four value stream partners described in this paper, are described in the following section drawing the reader's attention to important lessons learned from implementing concepts introduced during the LL Program, challenges faced, and specific examples captured during the visits.

\section{FOUR STORIES OF THE VALUE STREAM PARTNERS}

\section{STORY 1: Digital FABRication}

A prefabrication company, which started in 2016, uses digital designs to prefabricate panelized structures and exterior and interior wall panels. They have recently added finished exterior wall assemblies and Mechanical Electrical and Plumbing (MEP) roughin to prefabbed wall panels. The company, which has grown to over one hundred twenty people, is now providing panels to projects across the United States.

The rapid growth of this company fostered a community of learning with effective knowledge transfer and team collaboration, which led to a plant-wide training focused on Lean principles. In addition to the plant-wide training, the company's leadership group enrolled in an LL course to continue to strengthen their Lean culture. A recent graduate mentioned how the courses reset the effectiveness of company meetings by specifically focusing teams on action-oriented items. Another student, who works on the shop floor, observed how "go and see" helps create an organic interaction between the design team and fabrication staff.

During the site visit, attended by authors one and two, a discussion ensued between the Lean Integrator and students where students shared what Lean principles and tools were in place and what future Lean goals they had. They indicated the following:

- Weekly Lean learning takes place and future Lean leadership will take place.

- Visual management is in place throughout the shop including the tracking of defects and production as shown below. Soon, they expect that a new system will be online to provide daily production feedback for real-time adjustments. Figure 2a illustrates boards that the foreman uses to review visual metrics with their line workers each week to learn and improve. 


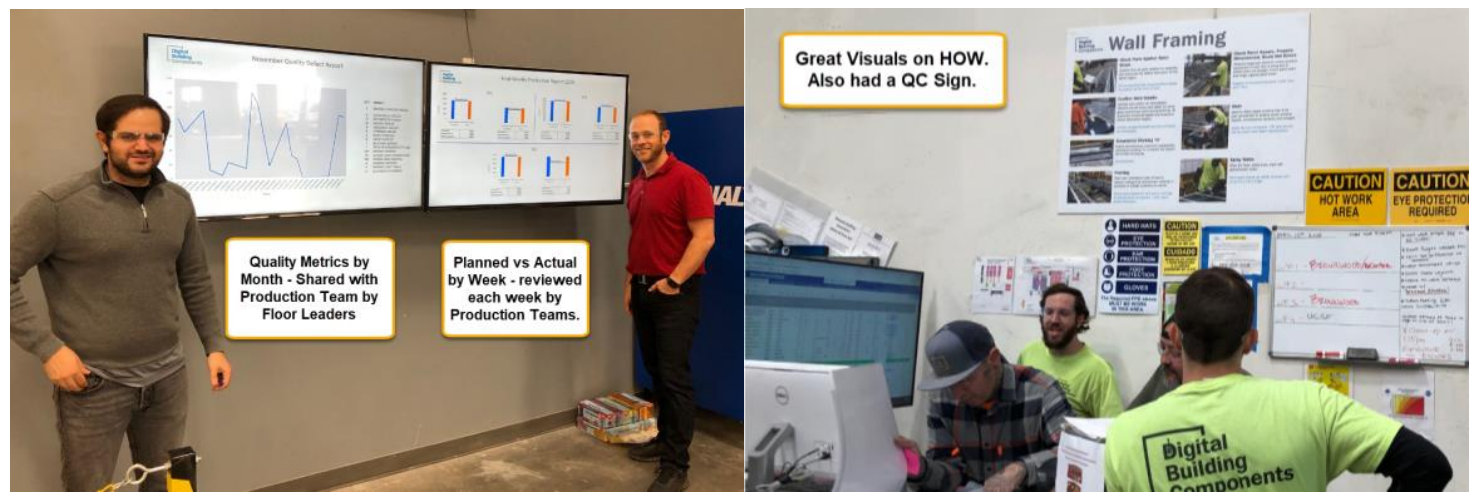

Figure 2: (a) Visual management of production and defects; (b) Visual management of wall framing steps.

- Shop workers use a program called SmartSheet to see their workflow and production rates, which gives them real-time feedback. These activities originated from the plan developed in the scheduling software Primavera. The shop is now overlaying multiple projects in their schedule to see workflow stacking to level out production. The schedule is resource-loaded to manage mura (un-unevenness) and muri (overburden) in regards to the multiple projects fabricated in the shop.

- Fabrication lines are arranged for the best flow of material, including the panels to complete and those to be loaded onto a truck. Lines are also arranged by wall or floor type.

This rapidly growing company is using Lean principles to solve challenges they are facing, including:

- Accept the current state as bad as it may be. For example, the model often needs more work to digitally fabricate from. The company is educating its customers that the earlier they start with the model, the smoother the process will go.

- Aligning project team schedule adjustments with the fab shop to avoid double handling. A new role of Integration Manager has been added to stabilize this process.

- The Integration Manager will also help educate the preconstruction teams to understand site logistics and key installation factors to price the plan accurately.

- Truck availability and accuracy of orders delivered to site to avoid double handling. Working with key truckers and educating them is the goal.

As the Lean Integrator said, "Everyone must understand the vision of where we are going. I think that vision is ever forward and found in Lean principles." In addition to addressing the challenges presented above, the Integration Manager also assists with breaking down silos across all projects. For example, rethinking how teams build the project including installation methods, earlier buyout and procurement, and more upfront design and modelling to deliver a faster schedule with higher quality at the best overall cost, as it is discussed in case 4 . The ability to see the entire value stream throughout projects and how one step of the process affects the next is closely related to what Ballard and Howell (2003) define as the Lean Project Delivery System (LPDS). Specifically, the ability to jointly 
analyze product and process options puts in practice another important piece of the LPDS: work structuring, which addresses project definition, Lean Design, Lean Supply, and Lean assembly.

\section{Story 2: VDC \& Project Controls}

Company leaders realized that project teams had been staffed mostly with people new to the company and that the transfer of knowledge and culture had been difficult due to their rapid growth. The leaders created the vision of a strategic partner that could help support projects from Virtual Design and Construction to Project Controls. This new company started five years ago and now has over one hundred forty people. The company is like a "Big Room" because they focus day in and day out on certain tasks and guide projects scattered across the world to use best practices and improve them. Examples of standard processes that they apply to multiple projects includes modelling, slip sheeting (i.e., updating construction documents according to the latest revisions), RFI management, submittal registers creation, and change order administration.

A few of the strategic partner employees, who participated in the LL training last year, recommended the course to others. The course is structured in a way that encourages project teams to uncover more about each other in a professional capacity and the challenges they face within their workplace. During the Leader Standard Work lesson (i.e., turning non-routine work and non-value-creating wrok into value-creating routing work, etc.), another student shared a visual on how they track time per project and task. The visuals and weekly review result in team utilization improvement by ten percent. This process thinking approach helps the teams think of new ways to track production while also making their project teams more efficient. These successes have led the company to enrolling thirty people in the LL training in 2019.

Some of the challenges that this strategic partner is currently facing includes:

- Working with GC teams to create processes to successfully guide employees, management systems, and work flow, including digital slip sheeting, RFI's, change orders, and VDC.

- Coordination and communication issues due to the remote location of the strategic team.

The common language and training that LL offers speeds up alignment, bringing about process improvements. As a result, the integration occurring between the GC team and the strategic partner creates a baseline of standard procedures that are used on multiple projects across the country. They have determined this to be the most successful model to accelerate knowledge transfer on standard procedures and improve them.

\section{STORY 3: EQUIPMENT RENTAL AND SUPPLIES}

This retail company is one of the industry leaders in providing equipment and supplies for construction job sites throughout the United States. In the last five years, they have doubled in size and now have eighteen locations throughout the United States.

A few employees new to the company recently completed the LL training in order to better integrate into the company's culture. During the value stream mapping lesson, a student decided to undertake an analysis of the invoicing process and found that half of the 
process steps are waste. After the class, she re-evaluated the billing process and streamlined the steps by reducing invoicing time from three weeks to less than one week, eliminating many unnecessary steps. This also led to an increased cash flow as the cycle time was reduced from three to one.

During a site visit carried out by the first author, the students shared the progress of the Lean implementation that has taken place since the course:

- Visual management of orders that need to be filled each day. Added visual labels of all stock.

- Colour coding system ensuring equipment is ready to be procured (Figure 3).

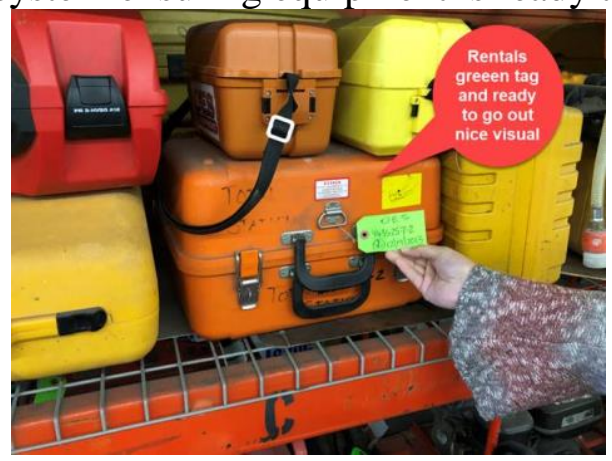

Figure 3: Equipment Rental Quality Control

During the training, the students who were new to the company began to see improvement opportunities based on the course content; some of which were implemented immediately and others which will be implemented in 2019. Additionally, the company's goal is to have every manager complete the course; this way everyone will understand the basics of Lean principles and how they can be applied in their work environment. Moreover, the team has become even more customer focused by working with project teams to determine what improvements need to be made. The GC is working to further their relationship with the retail company by purchasing big margin products to add value. In turn, the retail company can assist the GC with order efficiency and planning.

\section{Story 4: Large Construction Project}

The GC won a project to build a multi-use research facility using IPD delivery for a repeat customer. The owner, a sophisticated Lean advocate, wanted the team aligned around Lean principles. Furthermore, the project team leader considered study action groups and the reading of The Toyota Way to learn about and apply Lean principles. The first author suggested that the team enroll in the LL training instead of the study action groups. This was the first time an entire project team (i.e., contractor, key trade partners, owner, design team) enrolled in the course at the same time.

The class began with an overview of traditional thinking versus Lean thinking. Over the next nine weeks, the group also discussed thirteen lean principles and closed out with how to advocate lean thinking and mitigate resistance. Once the group completed the course, they discussed how and what to implement in their own project. The team's first step was to align everyone around a mission, vision, values, and operating principles, which gave participants a reference for how to operate within the big room environment set up 
for the project. Transitioning from design to construction, the team went into action using the idea of visual controls to better organize the team and problem solve. Some of the visual controls implemented included:

- A project website to share information in real-time. Project Cluster boards with team photos, goals for the week, and constraints. Eventually, these items migrated to online tools.

- BIM 360 Plan was used with the field crews to visually create and see the plan. The software also captures commitments to track Plan Percent Complete and reasons why activities were not complete. This real-time sharing of the plan and score were available for everyone to see, promoting a fly-wheel effect to propel the team to continuously improve their tasks.

- Dashboards of metrics like staff forecast and Request for Information (RFI) review time. Takt Planning for visual flow through the structure was implemented (Figure 5).

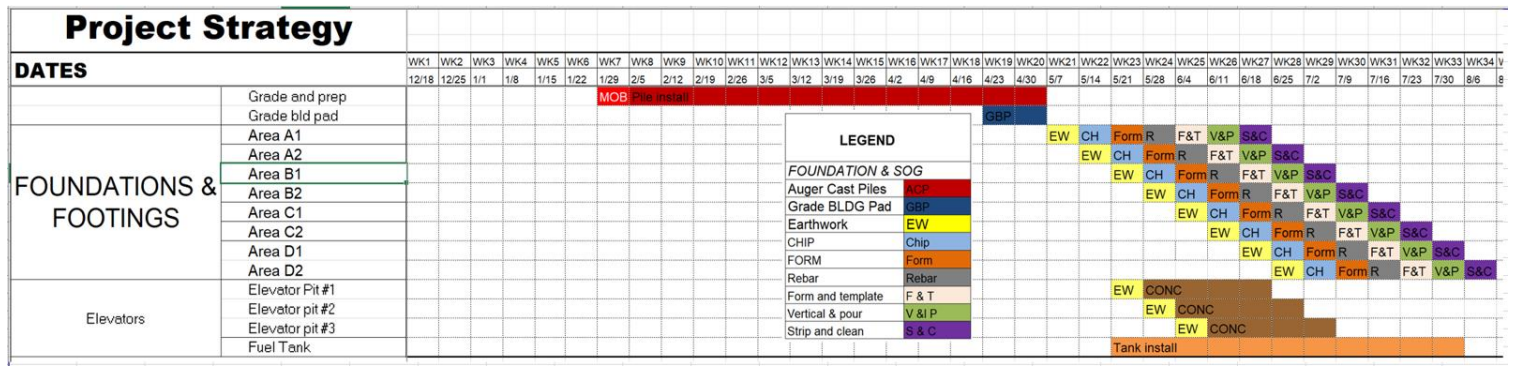

Figure 5: Takt Planning for Foundations

In addition, respect amongst employees drastically increased throughout this process. Rather than assume they knew what improvements were needed, management went directly to the field to discuss problems and possible solutions with the workers. Additionally, a scorecard was issued monthly to the extended team to allow them to grade key performance indicators (KPIs) and key behaviour indicators (KBIs). A KPI example is how often RFIs are answered on time while a KBI question is 'are you enjoying coming to work?' The GC often tracks KPIs but loses track of behaviours indicators in the process. When these indicators are grouped together, they address both performance and behavioural questions; the team then highlights the Lean principle 'respect for people'. Each month, the feedback received was analysed; typically, two to three themes, issues that needed attention, would become obvious. Once a theme was identified, it was sent out to the group to become the focus for the month. With a staff of around one hundred people, this communication became vital in creating a healthy environment.

Another major change for the project team was to implement prefabrication of all interior walls. They engaged the MEP and prefabricator early in the big room to coordinate their work together months before construction in the field would begin. This forced the coordination team to think differently, as they quickly realized that what worked in the past was not going to work on this project. The team decided that the most efficient installation would be to build partial height walls with posts rather than full height walls everywhere. 
This was reviewed with the design team and owner, who approved this solution, resulting in both cost and time savings.

The training inspired behaviour changes from the owner all the way to the employees in the field. For example, the design team tracked commitments and decisions that were needed to continue the value stream of design. The owner was more aware of the decisions that needed to be made and therefore, made them in a timely fashion. This enabled the project to stay on track. The KPI Scorecard allowed the team to make necessary changes, such as how to run effective meetings, and shed light on their commitment to work together to resolve issues. Tools such as KPIs encourage employees to drive changes and address the social needs of the team.

\section{DISCUSSION AND FUTURE WORK}

Since the piloting of the program in 2015, the Lean Leadership Course has continued to grow and gain momentum. After only three years, the national training gained such recognition that instructors were asked to offer a specific course to regional offices. This particular class, which began in 2018 , was and is supported by management, which enables even more employees to buy in and graduate. Instructors have even noticed a drastic increase in graduation numbers within the regional course, as the national classes sometimes experience a ten to twenty percent dropout rate. Additionally, three other regional groups are starting in 2019, including one from another strategic partner. The national group check-ins are all done via web meetings, while the specific regional groups are launched and concluded with a face-to-face meeting. This allows for a better connection between instructor and students, as it encourages better conversations from the start.

The instructors recognize the importance of continuing to evolve the course to better fit the needs of their students. As a result, they recently incorporated study action teams, which allows the students to reflect and participate in more meaningful discussions about the content. The 'training and apply your learning' method gives students a common language to use when problem solving, no matter their role or experience within the company. The instructors are also using the concept of train the trainers (multipliers). This has resulted in local graduates who are now teaching the LL course to additional business units, further tailoring the course content to meet the needs of the specific region. In turn, the new instructors become much more versed in the topics of LL.

Another Lean improvement is providing all the classes with a virtual classroom to collaborate, communicate, and coordinate with one another as well as the course content in between call-ins. The instructors found that e-mails often waste time, while virtual classroom technology allows students to maintain a running dialogue; one that is read and embellished upon at a much faster rate and in a more convenient forum. This also allows students to apply the concept of 'leader standard work' and utilize check-ins to ensure their group is keeping up with assignments. In addition, the digital classroom also allows students to hold each other accountable and become responsible for their own learning, i.e. teachers as well as learners. Undoubtedly, this creates a much more powerful learning experience, as they are able to reach out to one another for questions and comments, as well as the instructors. 


\section{CONCLUSIONS}

Adding the company's strategic partners to the LL training has helped people bond together around one common goal. Participants of the LL training are being given the opportunity to see the world through lean lenses. The training enables employees, in different business units, to recognize the company's shared vision and work together to achieve it. As evidenced in the stories presented, the training also provided students with the knowledge necessary to analyse, question, collaborate, and redesign their processes to improve flow of information and work through the entire value stream. These partners are writing their own story on how they are using the LL training to improve their businesses.

Moving forward, the company expects to: keep expanding opportunities for other partners in its extended value stream to participate in the LL training; align the partners and promote behaviours that can be easily replicated which improve quality, schedule, cost, and safety throughout the value stream; promote transparency by breaking down silo behaviours and sharing KPIs and KBIs to drive improvements and respect for the individual. These expectations represent additional opportunities to conduct research and identify specific KPIs and KBIs that will allow the company to evaluate how the changes are supporting its goals. Also, research on construction supply chains can be conducted to benchmark what specific actions deployed by these GC can be transferred to other construction organizations. Conclusions are limited to the value stream of this North American GC and the fact that the GC has a stake in these value stream partners.

\section{ACKNOWLEDGMENTS}

The authors would like to acknowledge the efforts of those who have participated in the training and helped redefine this journey. The views, comments, and opinions expressed in this paper are those of the authors and do not represent those of the company.

\section{REFERENCES}

Ballard, G. and Howell, G. (2003). Lean project management. Building Research \& Information. 31(2), 119-133.

Hackler C., Byse E., Reed D., and Alves, T.C.L. (2017). "Building a Lean Culture" In: LC3 2017 Volume II - Proc. of the 25 ${ }^{\text {th }}$ IGLC, Heraklion, Greece, 309-316.

Hackler C., Byse E., Alves, T.C.L., Reed D., (2018). "Lean Leadership training: lessons from a learner-centered analysis" In: Proc. of the 26th IGLC, Chennai, India, 484-494.

Rother, M. and Shook, J. (1998). Learning to See: Value Stream Mapping to Create Value and Eliminate Muda. Brookline, MA: Lean Enterprise Institute. 96pp.

Spencley, R., Pfeffer, G., Gordon, E., Hain, F., Reed, D., and Marosszeky, M. (2018). "Behavior-Based Quality Closing the Knowing Doing Gap." In: Proc. 26th IGLC, Chennai, India. 1170-1181

Tommelein, I.D., Walsh, K.D., Hershauer, J.C. (2003). Improving capital projects supply chain performance. CII RR 172-11. The University of Texas at Austin, 257pp.

Vrijhoef, R. and Koskela, L. (2000). "The Four roles of supply chain management in construction." European J. of Purchasing \& Supply Mgmt., 6, p. 169-178. 